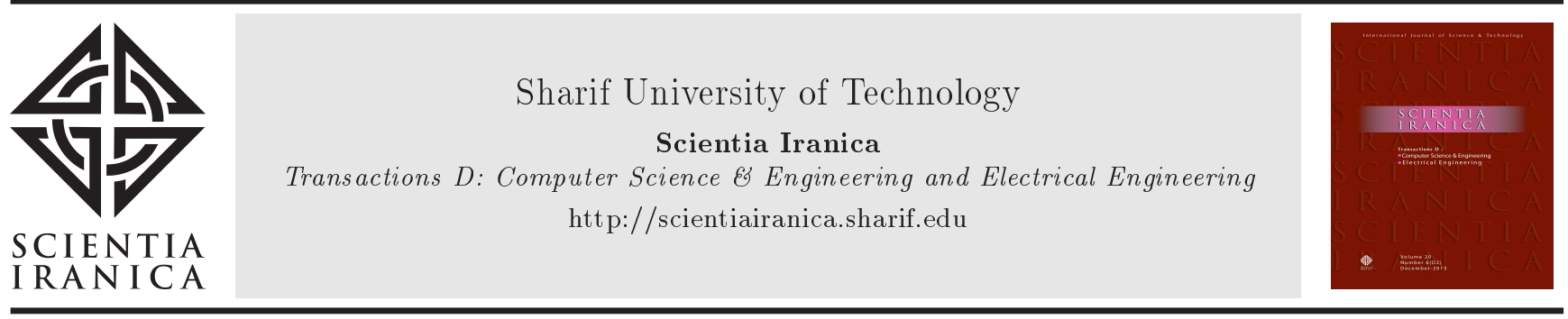

\title{
A novel inductive tactile probe design for lump detection in soft tissue phantoms
}

\author{
M.Z. Yildiz ${ }^{\mathrm{a}, *}$, H. Asadi Dereshgi ${ }^{\mathrm{b}}$, and A.F. Kamanli ${ }^{\mathrm{a}}$ \\ a. Department of Electrical and Electronics Engineering, Faculty of Technology, Sakarya University of Applied Sciences, Sakarya, \\ Sakarya, Turkey. \\ b. Mechatronics Engineering, Institute of Natural Sciences, Sakarya University, Sakarya, Turkey.
}

Received 27 May 2018; received in revised form 11 October 2018; accepted 22 December 2018

\author{
KEYWORDS \\ Tactile imaging probe; \\ Inductive tactile \\ sensor; \\ Human palpation; \\ Lump detection; \\ Psychophysics.
}

\begin{abstract}
The purpose of this study is the development of a novel tactile probe for detection of lumps in soft tissues. The tactile probe includes an inductive sensor inside an artificial tissue-like silicone rubber cushioning and a square shaped multi-metal array to increase sensitivity. First, a thermoplastic polymer probe was fabricated for housing and the mechanical compression test was executed by material testing machine. Second, the tactile sensor was calibrated between $2.5 \mathrm{~N}$ and $25 \mathrm{~N}$ within $2.5 \mathrm{~N}$ incremental steps and it showed $99.49 \%$ linear behavior. In order to measure the performance of the probe, 16 cylindrical silicone phantoms were used in three different scenarios. Each phantom was embedded by hard plastic inclusions in different depths and sizes. Finally, human palpation experiments were conducted by 10 naive subjects for the same scenarios above. The comparison results showed that, especially for deep inclusions at low forces, human subjects had high false diagnose rates, while the tactile sensor could detect the deep inclusions at all force levels (ANOVA, $p<0.001$ ). In summary, our novel tactile probe showed better performance than human palpation.
\end{abstract}

(C) 2020 Sharif University of Technology. All rights reserved.

\section{Introduction}

In recent years, according to the American Cancer Society Research (2018), breast cancer has been the first leading cause of cancer death in adult women around the world. However, when it is detected in the early stages, breast cancer can be cured with a high percentage of success. In 2018, according to the breast cancer statistics, almost 268, 670 new breast

\footnotetext{
*. Corresponding author. Tel.: +90 264 2957297;

Fax: +90 2642956424

E-mail addresses: mustafayildiz@sakarya.edu.tr(M.Z.

Yildiz); hamid.dereshgi@ogr.sakarya.edu.tr (H. Asadi

Dereshgi); fkamanli@sakarya.edu.tr (A.F. Kamanli)
}

doi: $10.24200 /$ sci.2018.51116.2013 cancer cases were reported in the United States among both genders [1]. Detection of breast cancer at its early stage of onset is crucial and highly dependent on the performance of the method used for breast cancer screening and diagnosis [2].

In the medical imaging literature, there are many ways to differentiate breast cancer tissue from healthy soft tissue. Mammography, ultrasonography, and Magnetic Resonance Imaging (MRI) play in important role in breast cancer detection [3-8]. These methods are considered as gold standard, yet they have some certain weaknesses, over-diagnosis rates, and side effects. Over-diagnosis may cause increase in the number of biopsies, higher rate of invasive procedures, psychological effects on a patient, and higher cost. Breast Self-Examination (BSE) and Clinical Breast Ex- 
amination (CBE) are also traditional human palpation methods, which mostly depend on the tactile sensitivity of human hand [9-12]. With the development of new tactile sensors, Tactile Imaging (TI) technologies have emerged [13,14].

$\mathrm{TI}$ is a non-invasive procedure adopted to detect cancerous tissues. It is used for mapping the interested area with sensors. In TI, every examiner can sense different shapes and geometries in different areas; hence, the results are quantitative and objective. Sensors with various types can detect the difference between the cancerous and healthy tissues [15-17]. By adjusting the TI size, the shape and depth of cancerous tissues can be detected. Geometric properties and stiffness vary between the benign and malignant tissues as reported in $[18,19]$. A limited number of devices are commercially available for TI applications [20]. Some groups of studies have dealt with different sensor types, namely accelerometers [21], piezoelectric polymers [22-25], magneto-inductance [26], ultrasonic technologies $[27,28]$, and sensor array with photo detector [29]. Although such sensors are used in a variety of applications, they mostly follow some fragile sensing mechanisms. Utilizing a product from Medical Tactile Inc., 2012, a study was conducted on 110 patients [30]. In another research, a silicone model was employed [31]. The research was carried out in two experimental steps first in linear motion and then, in circular motion if any problematic tissue was observed.

In the present study, we propose a novel hand-held single-point TI probe for automated palpation, which uses an updated inductive sensing element. The inductive sensing element was embedded in thermoplastic polymer housing to isolate the surrounding metal parts, which might cause electromagnetic noises. The housing was filled with a silicone rubber material and laser-cut metal array pieces placed $0.5 \mathrm{~cm}$ away from the sensing area to amplify the displacement sensitivity. The inductive element had $1 \mu \mathrm{m}$ displacement resolution. First, the tactile probe was designed by SolidWorks and created by a 3D printer. The silicone rubber part of the sensor used in this study was tested by a material compression testing machine. Moreover, the silicone rubber showed linear behavior in the selected force range, which was similar to the sensor output calibration curve. Silicone phantom models were also created to test the tactile probe under different scenarios. We hypothesized that our novel probe design would achieve a diagnosis rate with higher accuracy because of higher displacement resolution capabilities at static indentations. To test this hypothesis, we designed human and sensor experiments for comparative detection tasks. In order to detect the human diagnosis rate, we followed a method of constant psychophysical stimuli detection. The result was compared with the tactile probe performance by repeating the same procedure. The experimental results indicated that the TI sensor had better diagnosis rates for palpation than the human subjects, especially in small and deep inclusions.

\section{Materials and method}

\subsection{TI system design}

A tissue-like silicone rubber sensor was designed in a handy probe shape, as illustrated in Figure 1. Tactile probe dimensions and materials were selected in accordance with the manufacturing technology and the available materials on the market. The outer skeleton of the probe was produced from a thermoplastic polymer by a 3D printer. Commercial-grade silicone (Smooth-on Dragon Skin, USA) was used to construct a tissue-like cylindrical phantom. Silicone rubbers have numerous applications and one of their major characteristics is that they allow simulating tissue-like properties [32]. Our TI system consisted in

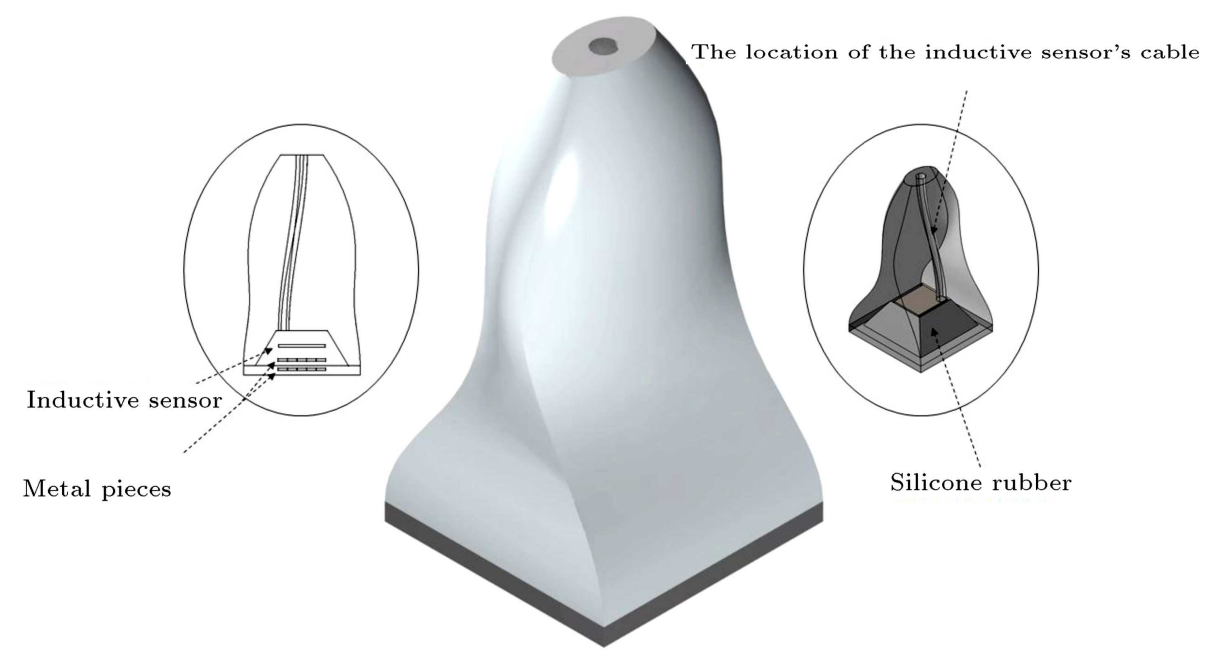

Figure 1. A schematics of the proposed tactile probe. 


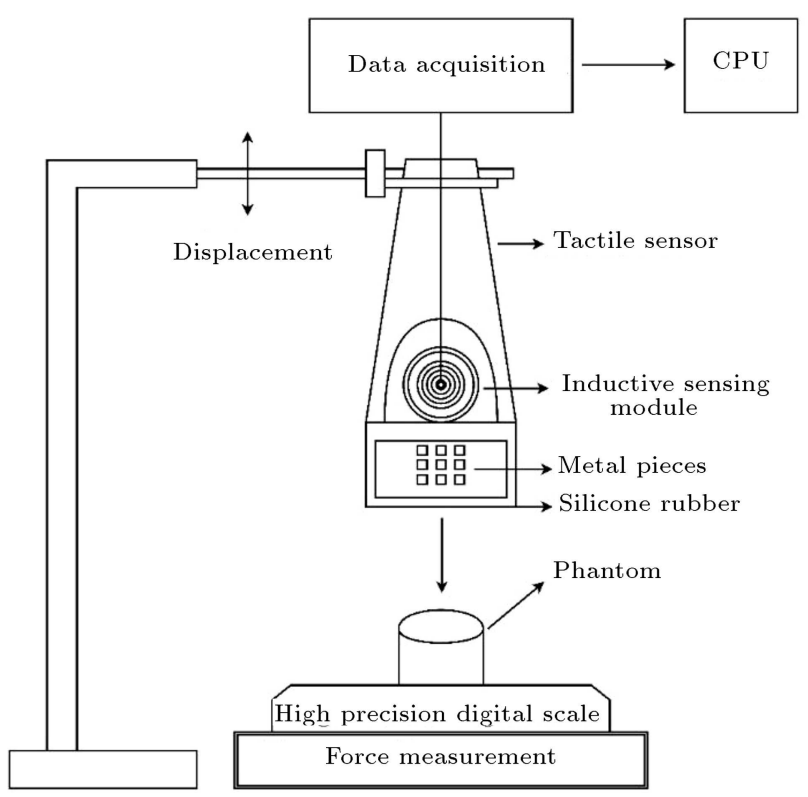

Figure 2. The setup for sensor calibration and tactile probe compression experiments.

Table 1. Characteristics of the tactile probe.

\begin{tabular}{ll}
\hline Tactile probe component & \multicolumn{1}{c}{ Specifications } \\
\hline Dimensions of probe & $50 \mathrm{~mm} \times 50 \mathrm{~mm} \times 94 \mathrm{~mm}$ \\
Dimensions of sensor & $16 \mathrm{~mm} \times 21 \mathrm{~mm} \times 1 \mathrm{~mm}$ \\
Touch section & $50 \mathrm{~mm} \times 50 \mathrm{~mm}$ \\
Dimensions of metal pieces & $5 \mathrm{~mm} \times 5 \mathrm{~mm} \times 1 \mathrm{~mm}$ \\
Thickness of silicone & $19 \mathrm{~mm}$ \\
\hline
\end{tabular}

inductive sensor elements (TI LDC1000 EVM, Texas Instruments, USA) arranged in silicone rubber cushioning. The TI sensor measured the parallel impedance of an inductor-capacitor network (LC) resonator and had an inductance-to-digital converter. This was made possible by energetic traces emitted by the resonator to regulate the oscillation amplitude in a closed-circuit configuration at a constant level. The sensor element was powered by an external $5 \mathrm{~V}$ DC regulated power supply. Inductive proximity sensors were used for contactless detection of metallic objects. In the designed probe, metal pieces were located on the top of the inductive sensor within the silicone embedding as shown in Figure 2. When a static indentation was applied to the probe surface, metal objects got closer to the inductive sensor, thereby changing the inductance value corresponding to surface displacements.

The system provided 24-bit inductance and displacement values. Beyond the distance of $10 \mathrm{~mm}$, the inductive sensor was not capable of sensing the outside metals, thereby reducing external noises. Some of the physical properties of the designed TI sensor are given in Table 1.

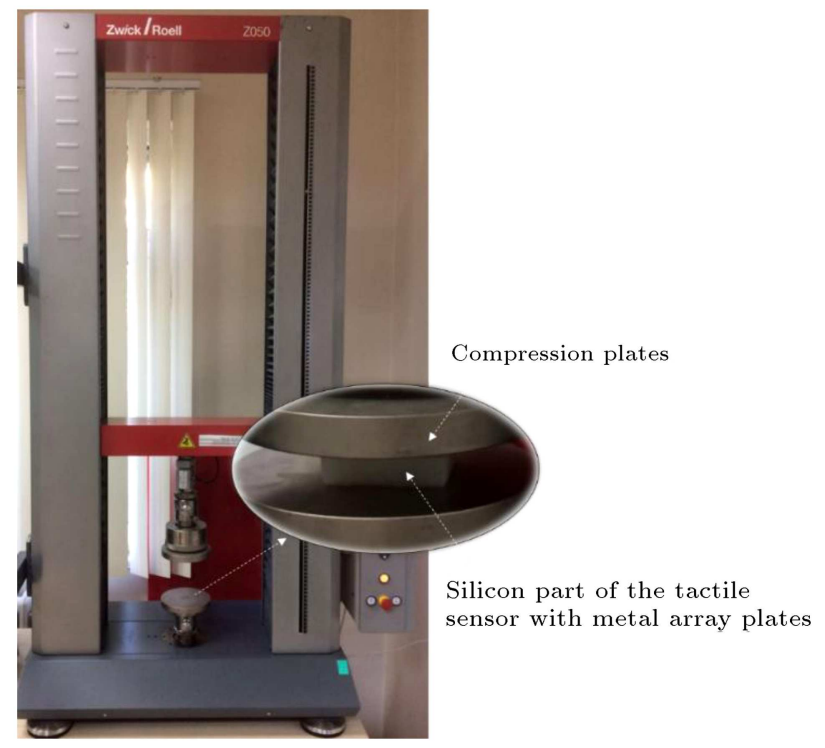

Figure 3. Compression test application to the silicone part of the sensor.
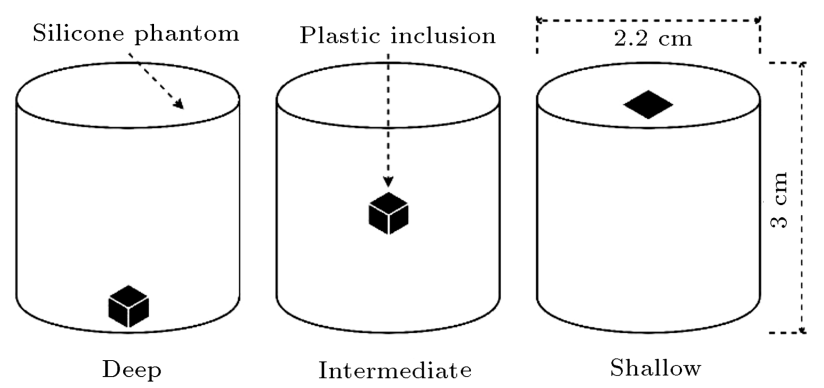

Figure 4. Locations of the hard-plastic inclusions in the silicone phantoms to mimic lumps in soft tissues.

\subsection{Compression test of the sensor}

The mechanical compression test of the tactile probe was executed by a material testing machine (Zwick Roell Z050) as shown in Figure 3. Force versus displacement values were recorded through a static compression test within the $0-25 \mathrm{~N}$ range at a speed of $2 \mathrm{~mm}$ per second. During the experiment, the silicone rubber part and the metal plates of the sensor were extracted from the thermoplastic outer surface in the design of the sensor. The results of the compression test are given in Section 3.

\subsection{TI probe experiment procedure}

We evaluated our probe design as shown in Figure 2. The tactile probe was attached to a fixed aluminum stand with hand movements along the $Y$-axis. The 3D printed probe included an inductive sensor from Texas Instruments embedded in silicone. In addition, each phantom had plastic inclusions at different locations as shown in Figure 4.

In the probe experiments, all phantoms were used at various forces. All phantoms had the same size 


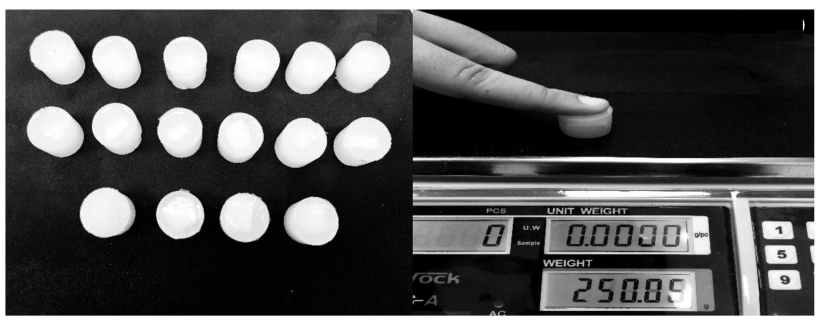

(a)

(b)

Figure 5. (a) Tissue-like silicone phantom models and (b) an example of human palpation experiments.

(height: $3 \mathrm{~cm}$, diameter: $2.2 \mathrm{~cm}$ ). A high-precision electronic scale was used to measure the applied forces and sensor responses were recorded as a function of force. The measurements were performed between $2.5 \mathrm{~N}$ and $25 \mathrm{~N}$ with the steps of $2.5 \mathrm{~N}$. The resultant data were used to obtain the calibration curve of the tactile sensor. Using the same experimental setup, we obtained TI probe output for different phantoms with inclusions of various sizes. Phantoms were embedded with plastic inclusions in different depths and sizes to mimic a lump in soft tissue, as shown in Figures 4 and $5(\mathrm{a})$.

\subsection{Human palpation procedure and psychophysical evaluation by yes/no method of constant stimuli}

In the signal detection theory, the observers are presented with various intensities. In our case, the intensity of the stimulus was considered as the size and location of the inclusions, as seen in Table 2. In each trial, phantoms were selected randomly by the operant and the task of the subject was to report whether there was an inclusion in the phantoms or not. The performance of human subjects was evaluated based on their false and correct responses. If there was an inclusion inside the phantom sample and the answer of the subject was 'YES', the response was considered correct and vice versa. During the experiments, all subjects used their right index finger tip.

First, subjects were trained for the empty control phantom (P.1) and 15 phantoms with inclusions. Then, they were blind folded and samples were randomly presented on a digital scale during the experiments. Every subject's responses were recorded and tabulated. The human palpation experiments were performed with 10 different volunteer human subjects (ages: 1924). Measurements were repeated for $5 \mathrm{~N}, 10 \mathrm{~N}$, and $15 \mathrm{~N}$ forces. Maintaining different static forces with only one finger is a difficult task for the human muscle system. Thereby, each subject was trained on a digital scale for three different forces as shown in Figure 5(b).

\subsection{Experimental cases}

Three different cases were employed to compare the sensitivities of the tactile probe and the human index finger. The primary task of the experiment were to see whether the tactile probe and human index finger could differentiate the empty phantoms from the phantoms having different size inclusions on the surface (Case 1), at the intermediate level (Case 2), and in deep point locations (Case 3). For each case, the phantoms were grouped as shown in Table 2.

\subsection{Statistical analyses}

Statistical analyses were performed in MATLAB 9.3. First, in TI probe experiments, 11 measurements were averaged. A two-way ANOVA was used to study the effect of force on displacement and size. Second, a multiple-comparison task was also carried out to see the effect of location of inclusions. Finally, in human experiments, the false diagnosis rates were calculated according to the signal detection theory.

\section{Results}

\subsection{Compression test results for the TI sensor}

Figure 6 shows the force-displacement relationship for the silicone part of the probe. As clearly shown, when the force was $25 \mathrm{~N}$, the silicone rubber material changed shape by $1.93 \mathrm{~mm}$. The experimental results showed linear behavior $\left(R^{2}=0.9942\right)$ between the applied

Table 2. The phantoms used in human palpation and tactile probe experiments.

\begin{tabular}{|c|c|c|c|c|c|c|c|}
\hline \multicolumn{2}{|c|}{ Phantom properties } & \multicolumn{2}{|c|}{ Case 1} & \multicolumn{2}{|c|}{ Case 2} & \multicolumn{2}{|c|}{ Case 3} \\
\hline Inclusion size & Element & $\begin{array}{c}\text { Inclusion } \\
\text { dimension } \\
\left(\mathrm{mm}^{2}\right)\end{array}$ & $\begin{array}{c}\text { Inclusion } \\
\text { depth }\end{array}$ & $\begin{array}{c}\text { Inclusion } \\
\text { dimension } \\
\left(\mathrm{mm}^{2}\right)\end{array}$ & $\begin{array}{c}\text { Inclusion } \\
\text { depth }\end{array}$ & $\begin{array}{l}\text { Inclusion } \\
\text { dimension } \\
\left(\mathrm{mm}^{2}\right)\end{array}$ & $\begin{array}{c}\text { Inclusion } \\
\text { depth }\end{array}$ \\
\hline- & P. 1 & Empty & - & Empty & - & Empty & - \\
\hline \multirow{2}{*}{ Small } & P. 2 & $2 \times 2$ & Surface & $2 \times 2$ & Intermediate & $2 \times 2$ & Deep \\
\hline & P. 3 & $3 \times 3$ & Surface & $3 \times 3$ & Intermediate & $3 \times 3$ & Deep \\
\hline \multirow{2}{*}{ Medium } & P. 4 & $5 \times 5$ & Surface & $5 \times 5$ & Intermediate & $5 \times 5$ & Deep \\
\hline & P. 5 & $5 \times 10$ & Surface & $5 \times 10$ & Intermediate & $5 \times 10$ & Deep \\
\hline Large & P. 6 & $5 \times 15$ & Surface & $5 \times 15$ & Intermediate & $5 \times 15$ & Deep \\
\hline
\end{tabular}




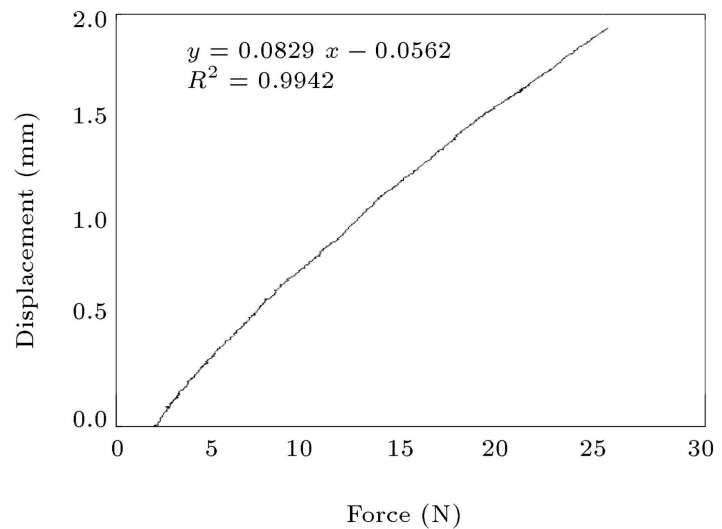

Figure 6. Surface displacement of the silicone part of the probe by the applied forces.

force value and the silicone surface. Accordingly, calculating the displacement with various force values became an easy task.

\subsection{TI probe calibration results}

In order to calibrate the TI sensor response, a forcedisplacement experiment was conducted. According the experimental results, the probe had a highly linear response $\left(R^{2}=99.49 \%\right)$, as shown in Figure 7 . The displacement values were differentiated around $8135 \mu \mathrm{m}$ and $8235 \mu \mathrm{m}(\Delta x=100 \mu \mathrm{m})$. The calibration curve showed similar linear characteristics to those in the mechanical compression test. The displacement values were not identical, as indicated in Figures 6 and 7 . The main reason might be that the displacement values in Figure 6 were measured by the compression test devices that gave the total shape difference on the $Y$-axis. However, the displacement values in Figure 7 belong to the metal pieces of the probe pressed on the phantom surface. Tanks to the submicron displacement resolution capability of the inductive sensor, these small values could be measured.

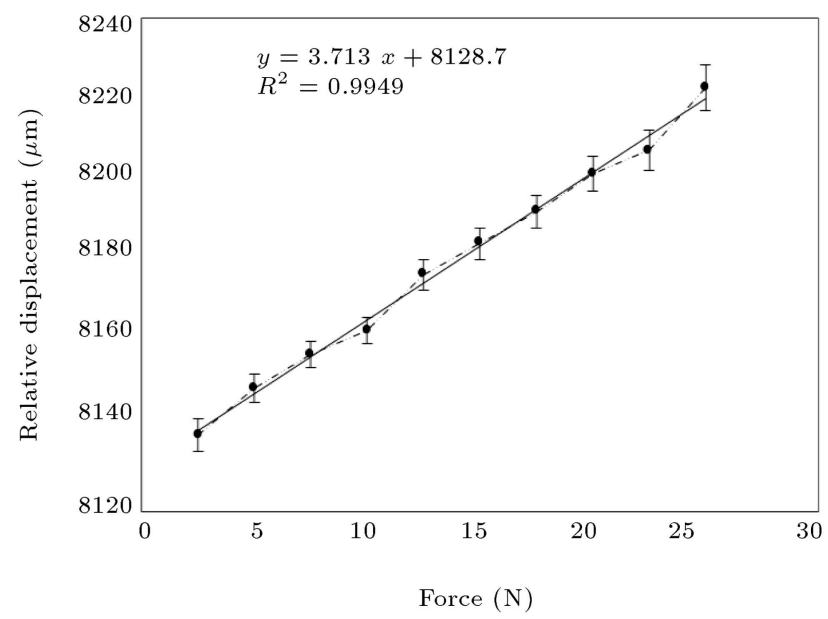

Figure 7. Relative displacement of sensor calibration as a function of force.

\subsection{TI probe experimental results}

After calibration, the TI sensor was used to test different scenarios with deep, intermediate, and surface inclusions for tissue-like silicone rubbers. Accordingly, three different cases were considered similar to those in Section 2.5.

First, the effects of the applied force on relative displacement values were plotted regardless of inclusion size, as seen in Figure 8. For all phantoms, the maximum and minimum displacement values were set to $15 \mathrm{~N}$ and $5 \mathrm{~N}$, respectively. A simple analysis of the main effects showed that increase in force level significantly enhanced the relative displacement values (ANOVA, $p<0.001$ ) for each case (i.e., surface, intermediate, and deep locations). Secondly, multiplecomparison test results showed that displacement values for the empty phantom at each force were significantly smaller than those for the deep, intermediate, and surface locations, regardless of inclusion size.

In Figure 9, the effect of inclusion size on the

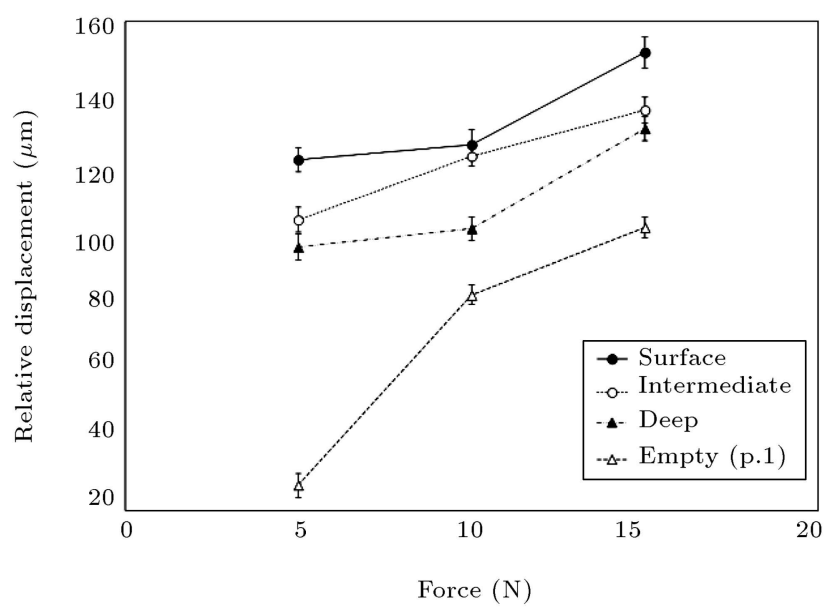

Figure 8. Relative displacement values of the tactile probe for Case 1: Surface inclusions, Case 2: Intermediate inclusions, and Case 3: Deep inclusions.

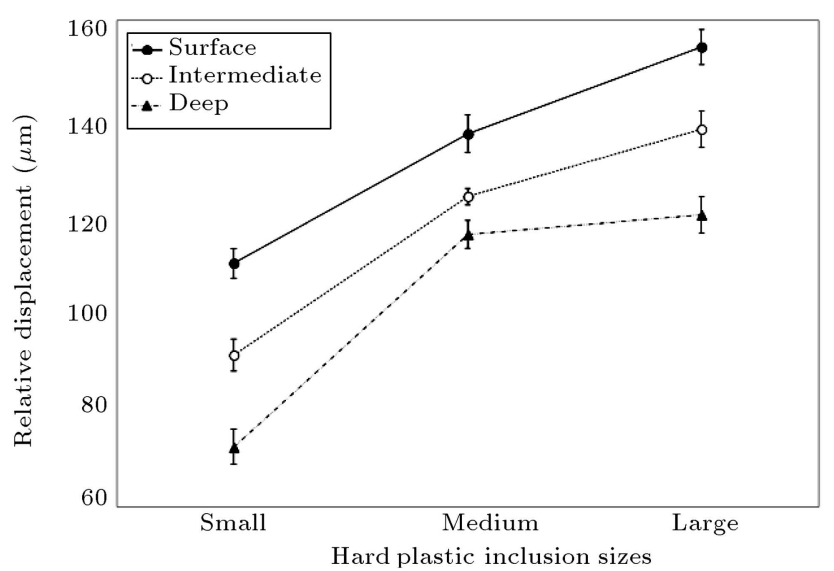

Figure 9. Relative displacement values of the tactile probe for small, medium, and large inclusions. 
value of relative displacement regardless of force level is shown. Statistical analysis indicated a significant interaction between the size and location of tumor-like silicone inclusions (ANOVA, $p<0.001$ ). Multiplecomparison analysis showed that large surface inclusions resulted in higher displacement values by the tactile probe and vice versa $(p<0.05)$. Accordingly, smaller displacement values made detecting low inclusions difficult.

\subsection{Human palpation experiments}

In human palpation experiments, the false diagnosis percentages of the subjects with respect to force, inclusion size, and location were recorded. Figure 10 shows the false diagnosis rates as a function of force level regardless of inclusion size. The maximum false diagnosis rates were measured with $5 \mathrm{~N}$ at deep inclusion locations. On the other hand, on the surface, the minimum rates were measured when the force was $15 \mathrm{~N}$. The effect of force on human detection was significant (ANOVA, $p<0.001$ ).

In human palpation experiments, in order to evaluate the effect of inclusion size with high precision, the false diagnosis rates at different forces were averaged and plotted as a function of relative size of inclusion. As indicated in Figure 11, it was observed that the false diagnoses made by the subjects increased as the depth of inclusions increased. On the other hand, regardless of inclusion size, false diagnosis rates remained almost constant at deeper locations (ANOVA, $p>0.05$ ). With human subjects, it was significantly more difficult to differentiate the size of the inclusions at deep locations. However, at surface and intermediate locations, false diagnosis rates were considerably affected by inclusion size (ANOVA, $p<0.05$ ). In these cases, the false diagnosis rates decreased with increase in the size of inclusion.

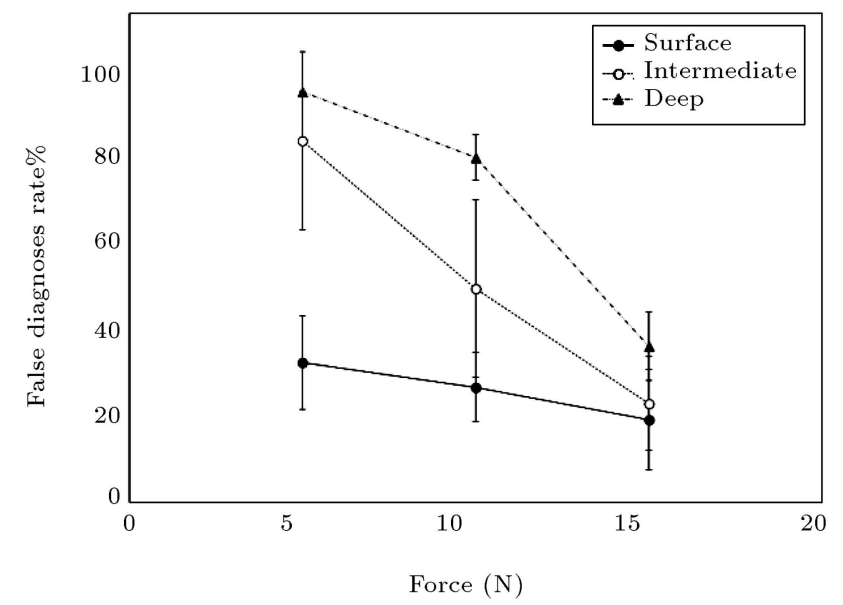

Figure 10. Human palpation false diagnosis rates regardless of inclusion size for Case 1: Surface inclusions, Case 2: Intermediate inclusions, and Case 3: Deep inclusions.

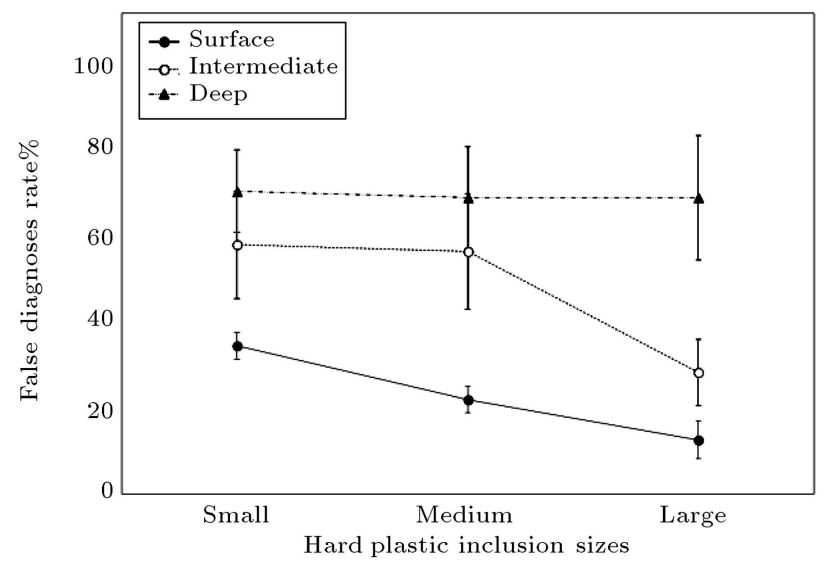

Figure 11. Human palpation false diagnosis rates regardless of the applied force values for small, medium, and large inclusions.

\section{Discussion}

In this study, we present a novel inductive TI probe, which might be helpful for tumor or lump detection in soft tissue. In our probe design, a single inductive sensor and a metal array were embedded into silicone rubber. The sensor was placed in the thermoplastic part to increase handling. The TI probe detected the local deformation on the silicone rubber surface, which caused the metal array get closer to the inductive sensor. By increasing the surface deformation, the sensor outputs also increased. The probe was calibrated on a digital scale and showed $99.49 \%$ linear behavior. Mechanical compression test results for the silicone, which also comprised the metal array, showed $99.42 \%$ linearity. Overall, a linear calibration of the probe was appropriate.

To investigate the performance of the TI sensor and compare it with human palpation, we conducted compression experiments on artificial silicone rubber phantoms. Each phantom contained hard thermoplastic materials pretending the tumorous part in the soft tissue. In the literature, the stiffness of the cancer tissue has been reported significantly higher than that of the surrounding tissue [33-35].

First, the TI sensor was compressed on the artificial phantoms containing surface, intermediate, and deep tumor-like inclusions at different forces. Then, human palpation experiments were conducted by 10 subjects on the same artificial phantoms. The inclusion detection capability of human index finger and the tactile probe were comparable, especially when the inclusion size was small or the inclusion was in a deep location, which was the most challenging situation. The results of the compression experiments showed that the TI probe could detect tumor-like inclusions embedded in artificial silicone rubber phantoms at every location and size.

In the literature, there are many tactile sensor 
Table 3. Different groups of studies that have designed tactile sensors for lump detection.

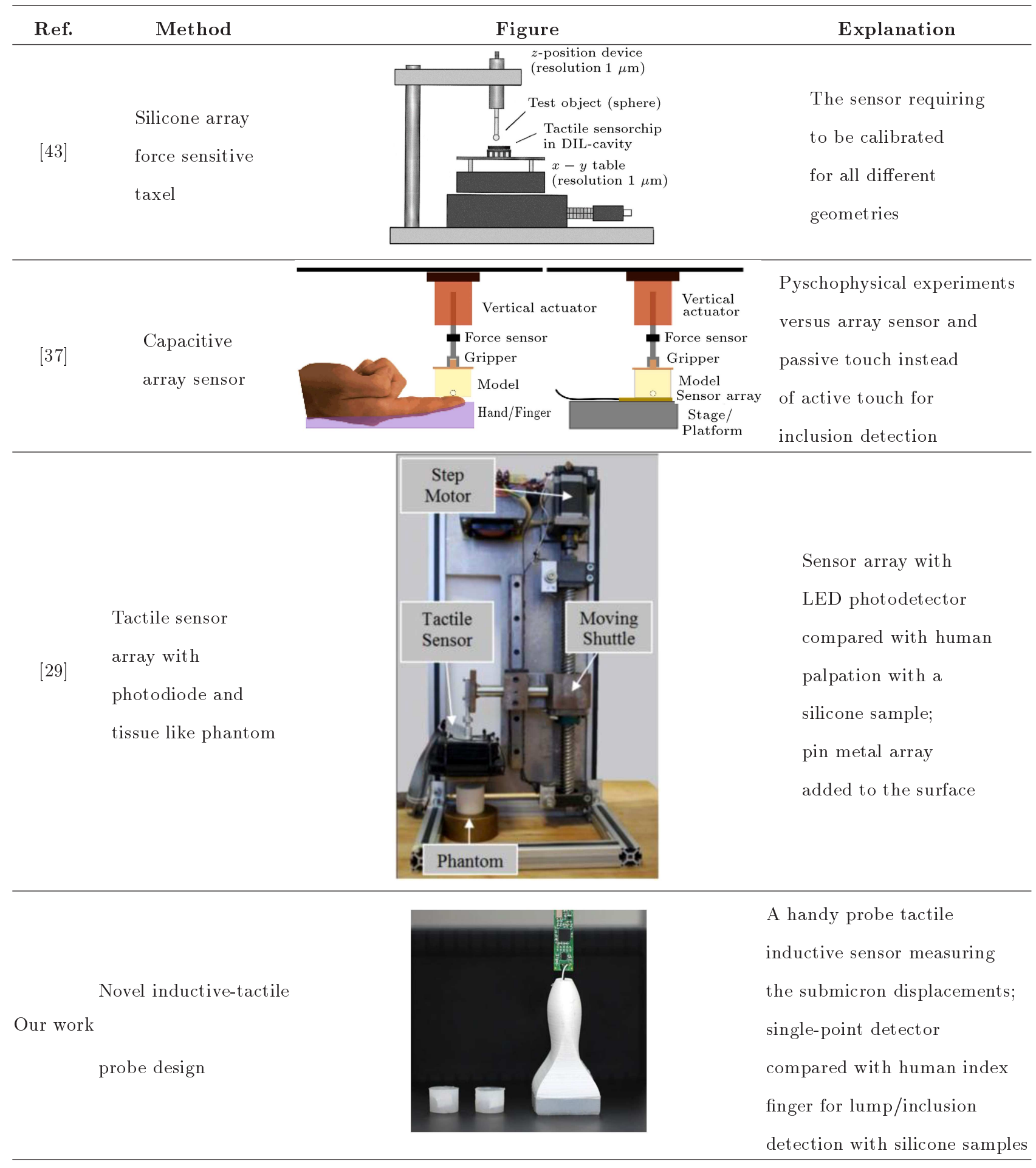

designs based on piezoresistive [36], capacitive [37], inductive [38], and optical $[39,40]$ sensors. Based on the fabrication technology and data acquisition circuitry, they can be classified as single [41] and array [42] types. Array-type tactile sensors are mostly used for shape recognition. They can be performed as robot-assisted palpation or manually by human hand. Single-point detection sensors can be used to collect information on depth, stiffness, and even shape by proper position mapping methodologies.

In Table 3, studies on tactile sensor design using similar phantom models are summarized. In the study by Leinweber et al. (2000) [43], a silicone covered array sensor was tested to detect a sphere object. However, 
their sensor needed to be calibrated for every different geometrical object. However, it was not found useful for use in object recognition in tactile tasks.

In the study by Gwilliam et al. (2010) [37], a capacitive array sensor was designed and compared with human index finger palpation capabilities utilizing artificial silicone samples. The samples had inclusions with different sizes in different depths. Their psychophysical aim was to find the minimum indentation depth and force required to detect an object in each silicone phantom. The sensor outputs at different indentation levels were also recorded. They showed that tactile sensor could make proper detection at lower indentation depths and pressures than human subjects.

In a similar study by Ayyildiz et al. (2013) [29], an optical tactile sensor was designed in array shape. The sensor was calibrated by a robot-assisted platform. Silicone samples with inclusions of different sizes were prepared. They also followed their psychophysical aim by active palpation and found that the tactile sensor could differentiate deeper and softer inclusions than human palpation. The biggest disadvantage of their study was that the surface of the sensor was covered by an array of metal pins, which were not proper for exerting pressure on the soft samples. The array shape, in addition to exact shape recognition, was used to compare the size of inclusions.

In the proposed probe design, the sensing area consisted in a single element, but there was a metal array embedded in the silicone cushioning. In this way, the surface deflections were amplified and inductance values were changed by the metal array pieces. The array sensors have high shape recognition capabilities. However, their signal processing time and cross-talk problems still need to be addressed. In the design of single-point-detection TI sensor, signal processing is faster without requiring any complex instrumentation or power supply systems. When the tumor is deeper and not eligible for manual palpation, the tactile probe cannot be used. Instead, other imaging modalities will be preferred.

The most challenging part of the inclusion detection task is when the phantom samples have deep and small inclusions. In a study by Gwilliam et al. (2010) [37], human subjects used their index fingers. Unlike our study, human palpation was executed by passive touch. In contrast, we chose active touch as the common approach to many palpation tasks. Human subjects had high false diagnosis rates, especially at low force levels, whereas the TI sensor was capable of differentiating the empty control phantoms from the inclusions at all depths. All in all, according to our experimental results, the proposed inductive TI probe might be one of the best candidates for inclusion detection in soft tissue phantoms.

\section{Conclusions}

In this study, we showed that inductive-based Tactile Imaging (TI) probe design could detect hard lumps in environments such as soft tissues with higher accuracy than a naive human subject in active palpation. The most difficult task in real life and the presented artificial scenarios in this study is finding deep and small-size inclusions in soft tissues. The samples were similar to those of the study by Ayyildiz et al. [29]. In the TI sensor compression experiment, even at the smallest forces, the statistical results showed that our TI probe could differentiate deep and small inclusions from the empty reference phantom. In all conditions, the sensor had superior performance.

In the future studies, the authors aim to design a TI imaging system that includes a high-resolution camera for positioning and collecting spatial information. Different psychophysical tasks will also be considered for naive and expert subjects for deep and more realistic comparisons.

\section{Acknowledgements}

This study was supported by Sakarya University Research Fund (BAP) no. 2014-01-00-001 to Assistant Professor Dr. Mustafa Zahid Yildiz. The authors thank professors from Mechatronics Engineering Department of Technology for their support and suggestions in the initial design of the experiments as well as their critical comments on the paper. The authors are also grateful to Biomedical Instrumentation Lab. Students for their contributions.

\section{References}

1. Siegel, R.L., Miller, K.D., and Jemal, A. "Cancer statistics", CA: A Cancer Journal for Clinicians, 68(1), pp. 7-30 (2018).

2. Bick, U. "Intensified surveillance for early detection of breast cancer in high-risk patients", Breast Care, 10(1), pp. 13-20 (2015).

3. Sung, J.S., Stamler, S., Brooks, J., et al. "Breast cancers detected at screening MR imaging and mammography in patients at high risk: method of detection reflects tumor histopathologic results", Radiology, 280(3), pp. 716-722 (2016).

4. Cosgrove, D.O., Kedar, R.P., Bamber, J.C., et al. "Breast diseases: color Doppler US in differential diagnosis", Radiology, 189(1), pp. 99-104 (1993).

5. Raza, S. and Baum, J.K. "Solid breast lesions: evaluation with power Doppler US", Radiology, 203(1), pp. 164-168 (1997).

6. Kuhl, C.K., Strobel, K., Bieling, H., et al. "Supplemental breast MR imaging screening of women with average risk of breast cancer", Radiology, $\mathbf{2 8 3}(2)$, pp. 361-370 (2017). 
7. Lashkari, A.E. and Firouzmand, M. "Developing a toolbox for clinical preliminary breast cancer detection in different views of thermogram images using a set of optimal supervised classifiers", Scientia Iranica, 25(3), pp. 1545-1560 (2018).

8. Quintanilla-Dominguez, J., Ojeda-Magaña, B., Cortina-Januchs, M.G., et al. "Image segmentation by fuzzy and possibilistic clustering algorithms for the identification of microcalcifications", Scientia Iranica, 18(3), pp. 580-589 (2011).

9. GüÇlü, B. and Bolanowski, S.J. "Vibrotactile thresholds of the Non-Pacinian I channel: II. Predicting the effects of contactor location on the phalanx", Somatosensory \& Motor Research, 22(1-2), pp. 57-68 (2005).

10. Bolanowski, J.R., Stanley, J., Verrillo, R.T., et al. "Four channels mediate the mechanical aspects of touch", The Journal of the Acoustical Society of America, 84(5), pp. 1680-1694 (1988).

11. Gescheider, G.A. "Psychophysical scaling", Annual Review of Psychology, 39(1), pp. 169-200 (1988).

12. Gescheider, G.A., Bolanowski, S.J., Pope, J.V., et al. "A four-channel analysis of the tactile sensitivity of the fingertip: frequency selectivity, spatial summation, and temporal summation", Somatosensory \& Motor Research, 19(2), pp. 114-124 (2002).

13. Yildiz, M.Z. and Güçlü, B. "Relationship between vibrotactile detection threshold in the Pacinian channel and complex mechanical modulus of the human glabrous skin", Somatosensory \& Motor Research, 30(1), pp. 37-47 (2013).

14. Gescheider, G.A., GüÇlü, B., Sexton, J.L., et al. "Spatial summation in the tactile sensory system: Probability summation and neural integration", Somatosensory \& Motor Research, 22(4), pp. 255-268 (2005).

15. Samani, A., Bishop, J., Luginbuhl, C., et al. "Measuring the elastic modulus of ex vivo small tissue samples", Physics in Medicine and Biology, 48(14), pp. $2183-2198$ (2003).

16. Wellman, P., Howe, R.D., Dalton, E., et al. "Breast tissue stiffness in compression is correlated to histological diagnosis", Technical Report, Harvard BioRobotics Laboratory, USA (1999).

17. Sarvazyan, A., Goukassian, D., Maevsky, E., et al. "Elastic imaging as a new modality of medical imaging for cancer detection", Proceedings of the International Workshop on Interaction of Ultrasound with Biological Media, Valenciennes, France, pp. 69-81 (1994).

18. Skovorda, A.R., Klishko, A.N., Gusakian, D.A., et al. "Quantitative analysis of mechanical characteristics of pathologically altered soft biological tissues", Biofizika, 40(6), pp. 1335-1340 (1995).
19. Krouskop, T.A., Wheeler, T.M., Kallel, F., et al. "Elastic moduli of breast and prostate tissues under compression", Ultrasonic Imaging, 20(4), pp. 260-274 (1998).

20. Tiwana, M.I., Redmond, S.J., and Lovell, N.H. "A review of tactile sensing technologies with applications in biomedical engineering", Sensors and Actuators A: Physical, 179, pp. 17-31 (2012).

21. Howe, R.D. and Cutkosky, M.R. "Sensing skin acceleration for slip and texture perception", IEEE Int. Conf. on Robotics and Automation, pp. 145-150 (1989).

22. Dario, P., De Rossi, D., Domenici, C., et al. "Ferroelectric polymer tactile sensors with anthropomorphic features", IEEE Int. Conf. on Robotics and Automation, pp. 332-340 (1984).

23. Son, J.S., Monteverde, E.A., and Howe, R.D. "A tactile sensor for localizing transient events in manipulation", IEEE Int. Conf. on Robotics and Automation, pp. 471-476 (1994).

24. Yamada, Y. and Cutkosky, M.R. "Tactile sensor with 3 -axis force and vibration sensing functions and its application to detect rotational slip", IEEE Int. Conf. on Robotics and Automation, pp. 3550-3557 (1994).

25. Dario, P., Rucci, M., Guadagnini, C., et al. "An investigation on a robot system for disassembly automation", IEEE Int. Conf. on Robotics and Automation, pp. 3515-3521 (1994).

26. Vranish, J. "Magnetoinductive skin for robots", IEEE Int. Conf. on Robotics and Automation, pp. 1292-1318 (1986).

27. Hutchings, B.L., Grahn, A.R., and Petersen, R.J. "Multiple-layer cross-field ultrasonic tactile sensor", IEEE Int. Conf. on Robotics and Automation, pp. 2522-2528 (1994).

28. Ando, S. and Shinoda, H. "Ultrasonic emission tactile sensing", IEEE Control Systems Magazine, 15(1), pp. 61-69 (1995).

29. Ayyildiz, M., Guclu, B., Yildiz, M.Z., et al. "An optoelectromechanical tactile sensor for detection of breast lumps", IEEE Transactions on Haptics, 6(2), pp. $145-155$ (2013).

30. Egorov, V. and Sarvazyan, A.P. "Mechanical imaging of the breast", IEEE Transactions on Medical Imaging, 27(9), pp. $1275-1287$ (2008).

31. Kaufman, C.S., Jacobson, L., Bachman, B.A., et al. "Digital documentation of the physical examination: Moving the clinical breast exam to the electronic medical record", The American Journal of Surgery, 192(4), pp. 444-449 (2006).

32. Martins, P.A.L.S., Natal Jorge, R.M., and Ferreira, A.J.M. "A comparative study of several material models for prediction of hyperelastic properties: Application to silicone-rubber and soft tissues", Strain, 42(3), pp. 135-147 (2006). 
33. Phipps, S., Yang, T.H.J., Habib, F.K., et al. "Measurement of tissue mechanical characteristics to distinguish between benign and malignant prostatic disease", Urology, 66(2), pp. 447-450 (2005).

34. Hoyt, K., Castaneda, B., Zhang, M., et al. "Tissue elasticity properties as biomarkers for prostate cancer", Cancer Biomarkers, 4(4-5), pp. 213-225 (2008).

35. Krouskop, T.A., Wheeler, T.M., Kallel, F., et al. "Elastic moduli of breast and prostate tissues under compression", Ultrasonic Imaging, 20(4), pp. 260-274 (1998).

36. Stassi, S., Cauda, V., Canavese, G., et al. "Flexible tactile sensing based on piezoresistive composites: A review", Sensors, 14(3), pp. 5296-5332 (2014).

37. Gwilliam, J.C., Pezzementi, Z., Jantho, E., et al. "Human vs. robotic tactile sensing: Detecting lumps in soft tissue", IEEE Haptics Symposium, pp. 21-28 (2010).

38. Wang, H., Kow, J., Raske, N., et al. "Robust and high-performance soft inductive tactile sensors based on the eddy-current effect", Sensors and Actuators A: Physical, 27(1), pp. 44-52 (2017).

39. Piacenza, P., Dang, W., Hannigan, E., et al. "Accurate contact localization and indentation depth prediction with an optics-based tactile sensor", IEEE Int. Conf. on Robotics and Automation (ICRA), Singapore, pp. 959-965 (2017).

40. Fujiwara, E., Wu, Y.T., dos Santos, M.F.M., et al. "Development of a tactile sensor based on optical fiber specklegram analysis and sensor data fusion technique", Sensors and Actuators A: Physical, 263, pp. 677-686 (2017).

41. Zou, L., Ge, C., Wang, Z.J., et al. "Novel tactile sensor technology and smart tactile sensing systems: A review", Sensors, 17(11), pp. 1-24 (2017).
42. Li, B., Shi, Y., Fontecchio, A., et al. "Mechanical imaging of soft tissues with a highly compliant tactile sensing array", IEEE Transactions on Biomedical Engineering, 65(3), pp. 687-697 (2018).

43. Leineweber, M., Pelz, G., Schmidt, M., et al. "New tactile sensor chip with silicone rubber cover", Sensors and Actuators A: Physical, 84(3), pp. 236-245 (2000).

\section{Biographies}

Mustafa Zahid Yildiz received the $\mathrm{PhD}$ degree in Biomedical Engineering from Bogazici University, Istanbul, Turkey, in 2013. He is currently a faculty member of Electrical and Electronics Engineering at Sakarya University of Applied Sciences, Sakarya, Turkey, and the director of the Biomedical Research Laboratory. His research interests are biomedical instrumentation, tactile psychophysics, and biomedical image processing.

Hamid Asadi Dereshgi received the MSc degree in Mechatronics Engineering from Islamic Azad University, Kashan, Iran, in 2013. He is currently a PhD candidate in Mechatronics Engineering, Institute of Natural Sciences at Sakarya University, Turkey. He is also a member of the Biomedical Research Laboratory at Sakarya University of Applied Sciences. His research interests are finite element modelling of materials, micro-electromechanical systems, microfluidics, and biomedical instrumentation.

Ali Furkan Kamanli received the MSc degree in Electrical and Electronics Engineering from Sakarya University, Sakarya, Turkey, in 2015. He is currently pursuing the $\mathrm{PhD}$ degree in Electrical and Electronics Engineering at Sakarya University. $\mathrm{He}$ is also a Research Assistant of the Biomedical Research Laboratory at the same university. His research interests are biomedical instrumentation, image processing, and medical electronics. 